\title{
Pathogenesis of human immunodeficiency virus-induced neurological disease
}

\author{
Andrew V Albright, Samantha S Soldan, and Francisco González-Scarano \\ Department of Neurology, University of Pennsylvania, Philadelphia, Pennsylvania, USA
}

\begin{abstract}
Infection of the central nervous system by the type 1 human immunodeficiency virus (HIV-1) commonly results in a number of neurological impairments known, in their most severe form, as HIV-associated dementia (HAD). The persistence of HIV encephalitis (HIVE), the pathological correlate of HAD, in spite of highly active antiretroviral therapy (HAART) underscores the importance of continued research focused on the neurobiology of HIV. To elucidate direct and indirect mechanisms of HIV neuropathogenesis, current investigation is focused on neuroinvasion, HIV-1-mediated mechanisms of neuronal damage and apoptosis, and compartmentalized evolution of virus in the brain. The aim of this review is to provide a selective overview of the most recent research on the neurobiology of HIV, adding only a brief introduction regarding established principles. Journal of NeuroVirology (2003) 9, 222-227.
\end{abstract}

Keywords: HIV; neuropathogenesis; entry

\section{Introduction}

In a significant subset of infected individuals, human immunodeficiency virus type 1 (HIV-1) infection is associated with a variety of neurological problems that are independent of any opportunistic infection. These include motor disturbances, cognitive impairments, and behavioral changes in various degrees of severity. The term HIV-associated dementia (HAD) is now used to describe this collection of neurological symptoms, particularly in its most severe form; minor cognitive motor disorder (MCMD) refers to milder versions of this problem, now more common since the advent of highly active antiretroviral therapy (HAART). In some patients, HAD is the initial manifestation of acquired immunodeficiency syndrome (AIDS); overall, it has been estimated to occur in $20 \%$ to $30 \%$ of untreated adults and in approximately half of pediatric cases (McArthur et al, 1993). HAART has reduced the incidence of HAD

Address correspondence to Francisco González-Scarano, Department of Neurology, Hospital of the University of Pennsylvania, 3400 Spruce Street, Philadelphia, PA 19104-4283, USA. E-mail: scarano@mail.med.upenn.edu

This work was supported by a grant from the American Foundation for AIDS Research (AmFAR) (to AVA) and by PHS grants NS-27405 and NS-07180 (to FG-S and SSS).

Received 9 January 2003; revised 13 January 2003; accepted 20 January 2003. by approximately 50\% (Maschke et al, 2000; Sacktor et al, 2002), but the frequency of HIV encephalitis (HIVE), its pathological manifestation usually characterized only in postmortem tissue, has remained constant (Masliah et al, 2000), suggesting that HAART does not eliminate HIV infection in the central nervous system (CNS), although HAART improves HIV infection symptoms quite dramatically. To better understand the pathological mechanisms of HAD and HIVE, current research is focused on HIV-1 entry into the (CNS), on the pathological and neurotoxic events that occur in response to CNS infection, and on the process of viral evolution within the CNS.

\section{HIV entry into the CNS}

HIV-1 enters the CNS early in the course of infection (Powderly, 2000), and the CNS is believed to remain a viral reservoir throughout the course of the infection, perhaps because of its relative immunological sequestration (Sinclair et al, 1994). The significant differences observed in nucleotide sequences and the biological properties of HIV-1 isolates from the brain and peripheral blood of the same patients suggest that the brain may harbor virus for years (Wong et al, 1997), as discussed in the last section of this review.

In order for HIV to enter a cell, it must bind to CD4, typically found on T lymphocytes, blood monocytes, macrophages, and some dendritic cells, 
and subsequently to one of a family of $\alpha$ or $\beta$ chemokine coreceptors. CCR5, the chemokine receptor for macrophage inflammatory protein (MIP)- $1 \alpha$ and MIP- $1 \beta$, is the primary coreceptor used by most HIV isolates recovered from the CNS, whereas CXCR4 is used by isolates in the periphery, particularly towards the later stages of AIDS. Viruses using CCR5 are referred to as R5 tropic, and those that use CXCR4 as X4 tropic (some strains are dualtropic, and can use either receptor). Within the CNS, HIV-1 infects mainly microglia, and monocytederived macrophages (Wiley et al, 1986). Because cells of the macrophage lineage are the only cells in the CNS that express both CD4 and CCR5, they are also the only productively infected cells within the brain (Bagasra et al, 1996). HIV-1 glycoproteinmediated syncytia formation, which results from fusion of microglia or brain macrophages, occurs in many HIV-infected cell types in vitro and may be observed as multinucleated giant cells (MNGCs) in situ. MNGCs are the defining feature of HIVE. In addition to microglia, other cells within the brain, including a subset of astrocytes expressing CXCR4, can be infected by HIV and harbor viral sequences. Although infection of blood-brain barrier (BBB) endothelial cells has been reported in some instances, there is no convincing evidence that endothelial cells are significant targets of HIV infection in humans (Edinger et al, 1997). However, because human brain microvascular endothelial cells express several of the cell surface glycoproteins involved in HIV-1 entry and infection (namely CXCR4, CCR5, and DC-SIGN and L-SIGN), a potential role for this cell type in HIV entry has not been ruled out (Mukhtar et al, 2002).

HIV is believed to enter the CNS either as free virus or via infected immune cells, crossing one of the physical barriers that protect and encase the CNS: the BBB and/or the cerebrospinal fluid (CSF)brain barrier. A likely source of the initial neuroinvasion is infected CD4+ lymphocytes, although it is also possible that cell-free virus penetrates the brain (Massari et al, 1990). Recent evidence suggests that cell-free HIV-1 particles may penetrate brain microvascular endothelial cells (BMVECs) through a mitogen-activated protein kinase (MAPK)-dependent macropinocytosis (Liu et al, 2002). BMVECs exposed to HIV-1 up-regulate expression of the intercellular adhesion molecule ICAM-1, which in turn may facilitate leukocyte migration across the BBB and increase the access of both cell-free HIV and infected monocytes/macrophages to the CNS (Liu et al, 2002). In addition, tumor necrosis factor (TNF)- $\alpha$, a proinflammatory cytokine secreted by infected macrophages (Mayne et al, 2000), increases BBB permeability by activation of guanylate cyclase and tyrosine kinase (Mayhan, 2002). Regardless of the potential role of cell-free virus and BMVECs, the most accepted model for entry of HIV into the CNS, also known as the "Trojan horse" hypothesis (Liu et al, 2000), suggests that HIV-1 enters the brain through the infiltration of infected monocytes that later differentiate into macrophages. Trojan horse transport of virus into the brain by infected monocytes and macrophages has also been described for visna virus, feline immunodeficiency viruses (FIV), simian immunodeficiency virus (SIV), and human T-cell leukemia virus type I (HTLV-I) (Georgsson et al, 1994; Romero et al, 2000), and it may therefore be a common mechanism for retroviral and lentiviral penetration of the brain. After crossing the BBB into the CNS, macrophages produce viral particles and spread productive HIV infection to neighboring microglia. The turnover rate of these perivascular microglia is relatively high compared to parenchymal microglia, which are long-lived (Kennedy and Abkowitz, 1997). A recent report differentiating between perivascular macrophages and parenchymal microglia based on the expression of myeloid markers suggests that the primary macrophage lineage cell type infected by SIV is the perivascular macrophage (Williams and Hickey, 2002). Similar results have been found for HIV infection. Regardless of the macrophage subtype infected in the CNS, all infected macrophages have the potential for secretion of neurotoxic factors.

The potential role of the CSF and the choroid plexus, the vascularized structure that makes up the boundary between the circulating blood and CSF, as a means for virus entry into the brain is a current area of research. The CSF functions as a partially separate compartment, and HIV strains derived from the choroid plexus are related to strains isolated from both the brain parenchyma and the periphery (Chen et al, 2002). Moreover, macrophages infected with some CSF isolates are able to mediate neuronal damage, possibly via apoptosis, as discussed later in this review (Chen et al, 2002). A recent study using the FIV model demonstrated productive infection of the choroid plexus and of macrophage-enriched choroid plexus cultures (Bragg et al, 2002), suggesting that the choroid plexus may be an important site for the trafficking of lentivirus into the CNS (Bragg et al, 2002). Other studies documented the infectability of choroids plexus cells, in vitro and in vivo (Petito et al, 1999; Harouse et al, 1989). Furthermore, if the CSF is seeded with more virulent $\mathrm{X} 4$ virus, direct neurotoxicity may occur if the CSF-brain barrier allows extracellular HIV particles to cross into the brain (Kaul and Lipton, 1999).

In addition to being a critical point for HIV entry into the CNS, the BBB and the CSF-brain barrier present physical impediments to the delivery of antiviral drugs. The therapeutic efficacy of HAART in the CNS is dependent at least in part upon its ability to achieve inhibitory concentrations. Protease inhibitors, which are eliminated from the CNS by the efflux transporters, P-glycoprotein and multidrug resistance-associated protein 1 , are less likely to achieve effective dose concentrations than other antiHIV pharmacologic compounds (Thomas et al, 2001). Additionally suboptimal concentrations of HAART 
in the CNS could lead to the development of drugresistant HIV strains. A recent report by Thomas and colleagues (2001) investigated the ability of abacavir, a nucleoside analog reverse transcriptase inhibitor, to cross these barriers and enter the brain in a guinea pig model. The authors found that abacavir can enter the brain at physiologically relevant concentrations, and is not inhibited by nucleoside transport inhibitors (Thomas et al, 2001). Conversely, it is reported that $98 \%$ of all drugs do not cross the BBB (Miller, 2002). Therefore, finding new means of allowing drugs to cross the BBB and gain access to the CNS is critical for treatment of HAD and elimination of the brain as a potential viral reservoir (Miller, 2002).

\section{Mechanisms of HIV-1-induced neurological disease}

There is no consensus on the underlying mechanisms of HIV-mediated neuropathogenesis, and therefore, it remains the subject of active research. Two predominant models have been proposed to explain the development of CNS abnormalities, both centering on the productive infection of brain macrophages and microglia. As mentioned previously, microglia and brain macrophages are the most frequently infected cell type of the CNS during all stages of infection and are the only productively infected cell type in the brain (Bagasra et al, 1996; Wiley et al, 1986; Koenig et al, 1986). They are believed to mediate neurological disease, either by producing neurotoxic viruses or viral proteins (Kaul and Lipton, 1999; Adamson et al, 1999; Johnston et al, 2001; Smith et al, 2001) or alternatively releasing endogenous compounds as part of the host response to virus infection (Kelder et al, 1998; McManus et al, 2000).

Viral proteins, including the HIV-1 envelope glycoprotein 160 (gp160), which is cleaved into two noncovalently associated products (gp120 and gp41), and the HIV transactivator protein, Tat, have the potential for neurotoxicity (Kaul and Lipton, 1999; Adamson et al, 1999; Johnston et al, 2001). Cleaved gp120 is soluble and can be shed from HIV-infected cells. At low concentrations, gp120 has been demonstrated to damage cultured neurons (Kaul and Lipton, 1999; Kanmogne et al, 2002). Although it was long suggested that gp120-mediated neuronal damage was achieved by a direct effect of gp120 on neurons, recent in vitro evidence suggests that gp120 neurotoxicity occurs indirectly and relies on the presence of toxic intermediates and activated chemokine receptors on macrophages/microglia. These toxic intermediates are thought to be inflammatory cytokines and arachidonic acid metabolites that are produced when macrophages or microglia are exposed to gp120 (Kaul and Lipton, 1999). An interesting model proposed that astrocytes, which express CXCR4, may also be involved in mediating such toxicity (Bezzi et al, 2001). This follows experiments that had indicated that ni- tric oxide synthase is induced in astrocytes exposed to gp120 (Nath, 1999). Nitric oxide production may impair the ability of astrocytes to protect neurons from damage. In vitro, neuronal toxicity and apoptosis has been demonstrated through the use of both $\mathrm{X} 4$ and dual-tropic envelopes, which is consistent with the finding that CXCR4 is present in a number of neural cell types (Kaul and Lipton, 1999; Gorry et al, 2002).

The neurotoxicity of HIV isolates is closely associated with the ability to induce fusion in monocytederived macrophages, which may result from the increased affinity for CCR5 exhibited by some HIV isolates of the CNS (Gorry et al, 2002). In vitro, this is indeed the case, as an isolate that was cultured for multiple rounds in microglia evolved into a highly fusogenic and pathogenic phenotype (Strizki et al, 1996). Further studies with that same passaged isolate indicated that specific amino acid changes correlate with increased fusion and decreased dependence on CD4 (Martín et al, 2001). Recently, CD4 binding of gp120 was demonstrated to induce a conformational change in the gp120 V1/V2 variable loops, which exposes the high affinity binding site for CCR5 (Kolchinsky et al, 2001; Zhang et al, 2001; Kwong et al, 2002). The loss of an $N$-linked glycosylation site in the V1/V2 stem appears to prevent occlusion of the CCR5-binding site and in some instances is sufficient for CD4-independent binding of gp120 to CCR5 (Kolchinsky, 2001). Therefore, the increased affinity for CCR5 exhibited by some neurotropic strains of HIV and SIV may result from changes in the V1/V2 stem. In addition, the CCR5-binding site of gp120 overlaps with an immunodominant epitope for neutralizing antibodies. Therefore, it has been postulated that in circumstances where the CCR5-binding site is exposed, the virus has an increased susceptibility to neutralization. One could then extrapolate that persistence of HIV/SIV strains with an increased affinity for CCR5 may result from the paucity of selective pressure exerted by neutralizing antibodies in the CNS, although this hypothesis is only now being addressed (Puffer et al, 2002).

The other cleavage product of gp160, gp41, was also shown to be neurotoxic in a study that demonstrated gp41 induction of nitric oxide production (Adamson et al, 1999). Tat is also secreted by infected cells and may induce neuronal death through apoptosis directly, via increases in intracellular calcium thereby stimulating the production of reactive oxygen intermediates and caspase activation; or indirectly, by stimulating macrophages to produce matrix metalloproteinases that induce neuronal apoptosis and whose expression is up-regulated in the brains of patients with HAD (Johnston et al, 2001). Recent evidence suggests that Tat toxicity is dependent upon a polyamine sensitive site on the $N$-methyl-D-aspartate receptor (Prendergast et al, 2002). In addition, it has been reported that both Tat and Nef increase production of neurotoxic quinolinic acid, a glutamate 
receptor agonist (Smith et al, 2001), whereas Vpr may cause apoptosis in human neurons (Patel et al, 2002). Although several HIV proteins have been reported to cause neurotoxicity, future studies should consider whether or not the concentrations of these viral proteins required for neurotoxicity in vitro are within the range present in vivo in infected brain.

Activated microglia, whether HIV infected or not, are important mediators of the innate immune system and are capable of producing a long list of soluble molecules and potential neurotoxins, including nitric oxide, superoxide anions, $\alpha$-chemokines, matrix metalloproteinases, glutamate receptor agonists, proinflammatory cytokines, growth factors, and superoxide anions (Bagasra et al, 1996, Gendelman et al, 1994). The neurotoxins produced by activated microglia have been implicated in several chronic progressive neurologic disorders, including HAD, Alzheimer's disease, Parkinson's disease, and multiple sclerosis. Levels of cytokine production, in particular interleukin (IL)-1, interferon (IFN)- $\gamma$, TNF- $\alpha$, tissue growth factor (TGF)- $\beta$, and IL-6, correlate roughly with the presence of HAD and may be secreted by macrophages, astrocytes, neurons, and endothelial cells when infected by HIV-1 or stimulated by activated macrophages (Kelder et al, 1998; Nath, 1999). Cytokines secreted by activated macrophages may result in bystander damage of both neurons and glia.

Ultimately, HIV-associated neurotoxic events, whether mediated by viral or cellular proteins, result in neuronal death, consistent with the symptomatology of HAD. Although there is little consistent evidence of neuronal infection in HIV infection, neuronal apoptosis can be detected in the brains of individuals with HAD, particularly in regions of the brain associated with neuropathological changes of MNGC formation. Similar changes have been noted in SIV encephalitis, the neuropathological outcome of SIV infection of rhesus macaques. Therefore, it has been postulated that neuronal apoptosis is the proximate cause of HIV-associated dementia. Two pathways of apoptosis have been proposed as potentially playing a role in HIV-mediated apoptosis: the extrinsic pathway is mediated by "death receptors" (TNF- $\alpha$ or fibroblast associated [FAS]), whereas the intrinsic pathway is mediated by mitochondria. The extrinsic and intrinsic pathways have common downstream partners, including activation of effector caspases, and they may engage in "crosstalk." In the intrinsic pathway, apoptosis is typically inhibited by the antiapoptosis Bcl2 family proteins. Although the predominant pathway involved in the neuronal apoptosis observed in HAD is unknown, it was recently shown that NMDA receptor/bcl-2regulated intrinsic apoptotic pathway contributes to neuronal apoptosis and that the Bcl-2 family of proteins protects neurons from the neurotoxic affects of HIV (Chen et al, 2002). Modulation of bcl-2 gene expression may confer neuroprotection and circumvent the development of HAD (Chen et al, 2002). Future research is focused on determining whether one or both of these pathways is involved with HIVD with the goal of blocking apoptosis in the brains of HIVinfected individuals, particularly if MCMD becomes a significant problem even in the face of adequate systemic therapy.

\section{Viral evolution}

As indicated previously in this review, HIV is known to penetrate the brain soon after the initial systemic infection. However, there is little evidence that primary viral seeding results in a permanently productive infection within the CNS. In fact, although the sampling error may be quite large, as most studies are performed at postmortem examination (i.e., after severe immunodeficiency has set in), viral RNA and antigens are infrequently detected during asymptomatic HIV infection (Gray et al, 1992). This is parallel to the findings in SIV infection, where there is minimal if any viral production until late in the course of simian AIDS (Williams et al, 2001). On the other hand, during the period of severe immunodeficiency (i.e., AIDS or SAIDS), viral replication in the CNS is quite robust, and strains with decreased dependence on CD4 and an increased sensitivity to neutralization can be isolated (Martin et al, 2001; Gorry et al, 2002; Puffer et al, 2002). The origin of this population indigenous to the CNS is still unclear; it may emerge as the result of waves of neuroinvasion during the course of the infection, or could be the result of low levels of replication in a quasilatent reservoir in relatively long-lived microglia. Activation of quiescently infected cells may provide low levels of ongoing replication and support adaptive evolution in the brain throughout infection. In addition, quasispecies may arise in the brain as a result of genetic drift of an isolated population or from selection or adaptation for growth in CNS cell types.

Recent work by our group examined the genomic SIV sequences found in individual SIV-infected MNGCs and compared these to the majority species present in brain and spleen (Ryzhova et al, 2002). The majority of genomic species present in the MNGCs were similar to the prevailing brain SIV genotype, indicating that at least a component of the population in this cell type comes from cells actively replicating virus. However, there was evidence of expression of archival genomes identical to the original inoculum in the region examined, indicating that some of the cells were seeded early during the course of infection. The isolation of archival sequences in the later stages of infection suggests that viral genomes remained in a latent, nonreplicating state of infection in at least a subpopulation of cells (Ryzhova et al, 2002). Latently infected microglia may support a low level of expression from this latent viral pool and contribute to compartmentalized virus evolution in the brain (Ryzhova et al, 2002). 


\section{Conclusions}

Although the advent of HAART has significantly prolonged and improved the lives of individuals living with HIV, the incidence of HIVE may be unchanged and systemic HIV infection is resistant to therapy in approximately $20 \%$ of patients participating in a HAART regimen. In addition, the vast majority of AIDS patients worldwide do not have

\section{References}

Adamson DC, Kopnisky KL, Dawson TM, Dawson VL (1999). Mechanisms and structural determinants of HIV-1 coat protein. Gp41 induced neurotoxicity. J Neurosci 19: $64-71$.

Ahlquist P (2002). RNA-dependent RNA polymerases, viruses and RNA silencing. Science 296: 1270-1273.

Bagasra O, Lavi E, Bobroski L, Khalili K, Pestaner JP, Tawadros R, Pomerantz RJ (1996). Cellular reservoirs of HIV-1 in the central nervous system of infected individuals: identification by the combination of in situ polymerase chain reaction and immunohistochemistry. AIDS 10: 573-585.

Bezzi P, Domercq M, Brambilla L, Galli R, Schols D, De Clercq E, Vescovi A, Bagetta G, Kollias G, Meldolesi J, Volterra A (2001). CXCR4-activated astrocyte glutamate release via TNF alpha: amplification by microglia triggers neurotoxicity. Nat Neurosci 4: 676-678.

Bragg DC, Childers TA, Tompkins MB, Tompkins W, Meeker RB (2002). Infection of the choroids plexus by feline immunodeficiency virus. J NeuroVirol 8: 211-224.

Chen W, Sulcove J, Frank I, Jaffer S, Ozdener H, Kolson DL (2002). Development of a human neuronal cell model for human immunodeficiency virus (HIV)infected macrophage-induced neurotoxicity: apoptosis induced by HIV type 1 primary isolates and evidence for involvement of the Bcl-2/Bcl-xL-sensitive intrinsic apoptosis pathway. J Virol 76: 9407-9419.

Edinger AL, Blanpain C, Kunstman KJ, Wolinsky SM, Parmentier M, Doms RW (1999). Functional dissection of CCR5 coreceptor function through the use of CD4independent simian immunodeficiency virus strains. J Virol 73: 62-73.

Gendelman HE, Lipton SA, Tardiru M, Bukrinsky MI, Nottet HSLM (1994). The neuropathogenesis of HIV-1 infection. J Leukoc Biol 56: 389-398.

Georgsson G (1994). Neuropathologic aspects of lentiviral infections. Ann N Y Acad Sci 724: 50-67.

Gorry PR, Taylor J, Holm GH, Mehle A, Morgan T, Cayabyab M, Farzan M, Wang H, Bell JE, Kunstmann K, Moore JP, Wolinsky SM, Gabuzda D (2002). Increased CCR5 affinity and reduced CCR5/CD4 dependence of a neurovirulent primary human immunodeficiency virus type 1 isolate. J Virol 76: 6277-6292.

Gray F, Lescs M, Keihane C, Paraire F, Marc B, Durigon M, Gherardi R (1992). Early brain changes in HIV infecton: neuropathological study of 11 HIV seropositive non-AIDS cases. J Neuropathol Exp Neurol 51: 177-185.

Harouse JM, Wroblewska Z, Laughlin MA, Hickey WF, Schonwetter BS, González-Scarano F (1989). Human choroid plexus cells can be latently infected with human immunodeficiency virus. Ann Neurol 25: 406-411. access to HAART (Powderly, 2000). Therefore, continued research focused on the neurobiology of HIV is imperative. An increased understanding of HIV infection and the pathogenesis of HAD and HIVE will be achieved by further research focused on neuroinvasion, HIV-mediated mechanisms of neuronal damage and apoptosis, antivirals with improved access to the CNS, and compartmentalized evolution of virus within the brain.

Johnston JB, Zhang K, Silva C, Shalinsky DR, Conant K, Ni W, Corbett D, Young VW, Power C (2001). HIV-1 Tat neurotoxicity is prevented by matrix metalloproteinase inhibitors. Ann Neurol 49: 230-241.

Kanmogne GD, Kennedy RC, Gramas P (2002). HIV-1 gp120 proteins and gp160 peptides are toxic to brain endothelial cells and neurons: possible pathway for HIV entry into the brain and HIV-associated dementia. $J \mathrm{Neu}$ ropathol Exp Neurol 61: 992-1000.

Kaul M, Lipton SA (1999). Chemokines and activated macrophages in HIV gp120-induced neuronal apoptosis. Proc Natl Acad Sci U S A 96: 8212-8216.

Kelder W, McArthur JC, Nance-Sproson T, McClernon D, Griffin DE (1998). $\beta$-Chemokines MCP-1 and RANTES are selectively increased in cerebrospinal fluid of patients with human immunodeficiency virus-associated dementia. Ann Neurol 44: 831-835.

Kennedy DW, Abkowitz JL (1997). Kinetics of central nervous system microglial and macrophage engraftment: analysis using a transgenic bone marrow transplantation model. Blood 90: 986-993.

Koenig S, Gendelman HE, Orenstein JM, Dal Canto MC, Pezeshkpour GH, Yungbluth M, Janotta F, Aksamit A, Martin MA, Fauci AS (1986). Detection of AIDS virus in macrophages in brain tissue from AIDS patients with encephalopathy. Science 233: 1089-1093.

Kolchinsky P, Kiprilov E, Bartley P, Rubinstein R, Sodroski J (2001). Loss of a single N-linked glycan allows CD4independent human immunodeficiency virus type 1 infection by altering the position of the gp120 V1/V2 variable loops. J Virol 75: 3435-3443.

Kwong PD, Doyle ML, Casper DJ, Cicala C, Leavitt SA, Majeed S, Steenbeke TD, Venturi M, Chaiken I, Fung M, Katinger H, Parren PWIH, Robinson J, Van Ryk D, Wang L, Burton DR, Freire E, Wyatt R, Sodroski J, Hendrickson WA, Arthos J (2002). HIV-1 evades antibody-mediated neutralization through conformational masking of receptor-binding sites. Nature 420: 678-682.

Liu NQ, Lossinsky AS, Popik W, Li X, Gujuluva C, Kriederman B, Roberts J, Pushkarsky T, Bukrinsky M, Witte M, Weinand M, Fiala M (2002). Human immunodeficiency virus type 1 enters brain microvascular endothelia by macropinocytosis dependent on lipid rafts and the mitogen-activated protein kinase signaling pathway. J Virol 76: 6689-6700.

Liu Y, Tang XP, McArthur JC, Scott J, Gartner S (2000). Analysis of human immunodeficiency virus type 1 gp160 sequences from a patient with HIV dementia: evidence for monocyte trafficking into brain. J NeuroVirol 6: S70S81. 
Martín J, LaBranche CC, González-Scarano F (2001). Differential CD4/CCR5 utilization, gp120 conformation and neutralization sensitivity between envelopes from a microglia-adapted human immunodeficiency virus type 1 and its parental isolate. $J$ Virol 75: 3568-3580.

Maschke M, Kastrup O, Esser S, Ross B, Hengge U, Hufnagel A (2000). Incidence and prevalence of neurological disorders associated with HIV since the introduction of highly active anti-retroviral therapy (HAART). J Neurol Neurosurg Psychiatry 69: 257-260.

Masliah E, DeTeresa RM, Mallory ME, Hansen LA (2000). Changes in pathological findings at autopsy in AIDS cases for the last 5 years. AIDS 14: 69-74.

Massari FE, Poli G, Schnitman SM, Psallidopoulous MC, Darvey V, Fauci AS (1990). In vivo T lymphocyte origin of macrophage-tropic strains of HIV. Role of monocytes during in vitro isolation and in vivo infection. JImmunol 144: 4628-4632.

Mayhan WG (2002). Cellular mechanisms by which tumor necrosis factor-alpha produces disruption of the bloodbrain barrier. Brain Res 927: 144-152.

Mayne M, Holden CP, Nath A, Geiger JD (2000). Release of calcium from inositol 1,4,5-triphosphate receptorregulated stores by HIV-1 Tat regulates TNF-alpha production in human macrophages. J Immunol 164: 65386542.

McArthur JC, Hoover DR, Bacellar H, et al (1993). Dementia in AIDS patients: incidence and risk factors. Neurology 43: 2245-2251.

McManus CM, Weidenheim K, Woodman SE, Nunez J, Hesselgesser J, Nath A, Berman JW (2000). Chemokine and chemokine-receptor expression in human glial elements: induction by the HIV protein Tat, and chemokine autoregulation. Am J Pathol 156: 1441-1453.

Miller G (2002). Breaking down barriers. Science 297: 1116-1118.

Mukhtar M, Harley S, Chen P, BouHamdan M, Patel C, Acheampong E, Pomerantz R (2002). Primary isolated human brain microvascular endothelial cells express diverse HIV/SIV-associated chemokine coreceptors and DC-SIGN and L-SIGN. Virology 297: 78-88.

Nath A (1999). Pathobiology of human immunodeficiency virus dementia. Semin Neurol 19: 113-127.

Patel CA, Mukhtar M, Harley S, Kulkosky J, Pomerantz RJ (2002). Lentiviral expression of HIV-1 Vpr induces apoptosis in human neurons. J NeuroVirol 8: 86-99.

Petito CK, Chen H, Mastri AR, Torres-Munoz J, Roberts B, Wood C (1999). HIV infection of choroid plexus in AIDS and asymptomatic HIV-infected patients suggests that the choroid plexus may be a reservoir of productive infection. J NeuroVirol 5: 670-677.

Powderly WG (2000). Current approaches to treatment for HIV-1 infection. J NeuroVirol 6(suppl 1): S8-S13.

Prendergast MA, Rogers DT, Mulholland PJ, Littleton JM, Wilkins LJ Jr, Self RL, Nath A (2002). Neurotoxic effects of the human immunodeficiency virust type-1 transcription factor Tat require function of a polyamine sensitivesite on the $N$-methyl-D-aspartate receptor. Brain Res 954: 300-307.

Puffer BA, Pohlmann S, Edinger AL, Carlin D, Sanchez MD, Reitter J, Watry DD, Fox HS, Desrosiers RC, Doms RW (2002). CD4 independence of simian immunodeficiency virus Envs is associated with macrophage tropism, neutralization sensitivity, and attenuated pathogenicity. J Virol 76: 2595-2605.

Romero IAM, Prevost MC, Perret E, Adamson P, Greenwood J, Couraud PO, Ozden S (2000). Interactions between brain endothelial cells and human T-cell leukemia virus type 1-infected lymphocytes: mechanisms of viral entry into the central nervous system. J Virol 74: 60216030.

Ryzhova EV, Crino P, Shawver L, Westmoreland SV, Lackner AA, González-Scarano F (2002). Simian immunodeficiency virus encephalitis: analysis of envelope sequences from individual brain multinucleated giant cells and tissue samples. Virology 297: 57-67.

Sacktor N, McDermott MP, Marder K, Schifitto G, Selnes OA, McArthur JC, Stern Y, Albert S, Palumbo D, Kieburtz K, De Marcaida JA, Cohen B, Epstein L (2002). HIVassociated cognitive impairment before and after the advent of combination therapy. J NeuroVirol 8: 136-142.

Sinclair E, Gray F, Ciardi A, Scaravilli F (1994). Immunohistochemical changes and PCR detection of HIV provirus DNA in brains of asymptomatic HIV positive patients. J Neuropath Exp Neurol 53: 43-50.

Smith DG, Guillemin GJ, Pemberton L, Kerr S, Nath A, Smythe GA, Brew BJ (2001). Quinolinic acid is produced by macrophages stimulated by platelet activating factor, Nef, and Tat. J NeuroVirol 7: 56-60.

Strizki JM, Albright AV, Sheng H, O’Connor M, Perrin L, González-Scarano F (1996). Infection of primary human microglia and monocyte-derived macrophages with human immunodeficiency virus type 1 isolates: evidence of differential tropism. J Virol 70: 7654-7662.

Thomas SA, Bye A, Segal MB (2001). Transport characteristics of the anti-human immunodeficiency virus nucleoside analog, abacavir, into brain and cerebrospinal fluid. J Pharm Exp Therapeu 298: 947-953.

Wiley CA, Schrier RD, Nelson JA, Lamper PW, Oldstone MBA (1986). Cellular localization of human immunodeficiency virus infection within the brains of acquired immune deficiency syndrome patients. Proc Natl Acad Sci U S A 83: 7089-7093.

Williams KC, Corey S, Westmoreland SV, Pauley D, Knight H, deBakker C, Alvarez X, Lackner AA (2001). Perivascular macrophages are the primary cell type productively infected by simian immunodeficiency virus in the brains of macaques: implications for the neuropathogenesis of AIDS. J Exp Med 193: 905-915.

Williams KC, Hickey WF (2002). Central nervous system damage, monocytes and macrophages, and neurological disorders in AIDS. Annu Rev Neurosci 25: 537-562.

Wong JK, Ignacio CC, Torriani F, Havlir D, Fitch NJ, Richman DD (1997). In vivo compartmentalization of human immunodeficiency virus: evidence from the examination of pol sequences from autopsy tissues. J Virol 71: 2059-2071.

Zhang PF, Bouma P, Park EJ, Margolick JB, Robinson JE, Zolla-Pazner S, Flora MN, Quinnan GV Jr (2002). A variable region 3 (V3) mutation determines a global neutralization phenotype and CD4-independent infectivity of a human immunodeficiency virus type 1 envelope associated with a broadly cross-reactive, primary virusneutralizing antibody response. Virol 76: 644-655. 